\title{
NO LIGHT DILATON IN GAUGE THEORIES
}

\author{
Bob HOLDOM and John TERNING \\ Department of Physics, University of Toronto, Toronto, Ontario, Canada MSS $1 \mathrm{~A} 7$
}

Recelved 22 October 1987

\begin{abstract}
We study the dynamical breakdown of approximate scale invariance in gauge theories with slowly varying couplings and without ultraviolet cutoff We ssolate the high energy contribution to the dilaton mass and obtain an expression for it in terms of the fermıon self-energy $\Sigma(k) \Sigma(k)$ is determined via an analytical treatment of the linearized, ladder Schwinger-Dyson equation We find that this contribution to the dilaton mass is large and is surprisingly insensitive to the $\beta$-function when the $\beta$-function is small. The result is that for a technicolor theory with a slowly varying coupling there is no light dilaton At most there is a massive scalar, the analog of the standard Higgs scalar, with a mass tied to the technifermion mass scale
\end{abstract}

There has been recent interest in chiral symmetry breaking occurring in theories having slowly varying gauge couplings. Old problems in technicolor theories concerning flavor changing neutral currents and low mass technipions may be alleviated $[1-4]$ and additional predictions may result as well [5]. A small $\beta$-function also gives some handle on the study of chiral symmetry breakıng beyond the ladder approximation [6].

A small $\beta$-function and chiral symmetry breakıng seem to imply a spontaneous breakdown of approximate scale symmetry. This led to speculation about a light dilaton in technicolor theories [3]. This particle is the analog of the standard neutral Higgs: it has the same quantum numbers and it couples similarly to quarks and leptons. But unlike the Higgs we may hope to determine the dilaton mass. The first guess is that the dilaton mass squared is proportional to the $\beta$-function, and could thus be small [3]. Another interpretation is that the dilaton mass is of order the confinement scale, which could also be small [7].

In ref. [8] we studied the contribution to the dilaton mass arising from energy scales large compared to the chiral symmetry breaking scale. In particular we isolated the contribution to the mass due to a physical ultraviolet cutoff, corresponding to new "sideways" physics in a technicolor theory. We found that this contribution in fact grew the slower the cou- pling $\alpha(k)$ fell for increasing $k$. When combined with the contribution arising from the variation of $\alpha(k)$ we found that the total high energy contribution to the mass was always large. A light dilaton was unlikely. (Ref. [9] continued the study of the dilaton mass for the case of a constant coupling, with a simlar conclusion, although the physical interpretation of the ultraviolet cutoff in that reference differs from ours.) But we found that the mass did decrease somewhat with increasing cutoff, and the possibility remained that a smaller mass could result if the sideways physics was represented by something less severe than a cutoff.

In this paper we ask how the dilaton mass behaves in a barely asymptotically free theory with no physical ultraviolet cutoff. We again focus on the high energy contribution to the dilaton mass. The slow variation of the coupling $\alpha(k)$ is the only source of explicit breakıng of scale invariance and one would guess that the slower $\alpha(k)$ decreased for increasing $k$, the smaller this contribution to the dilaton mass would become. But this is not what we find.

For the dilaton mass squared we derive an integral expression involving the fermion self-energy $\Sigma(k)$. The dependence on the $\beta$-function is such that if $\Sigma(k)$ were held fixed the mass squared would vanush linearly with $\beta$ as narvely expected. But previous work $[6,5]$ has shown that as $\beta$ decreases the function $k \Sigma(k)$ peaks at ever increasing momentum scales. 
The integrand in the mass formula behaves simularly and the net result is a dilaton mass with surprisingly little dependence on $\beta$. We obtain a simple expression for small $\beta$ which shows that the mass is of order of the dynamical fermion mass. This contribution may well domınate any further contribution to the dilaton mass arising from confinement physics.

This latter fact is well illustrated in a theory which generates a fermıon mass much higher than the confinement scale due to a large Casimir $C_{2}$ for the fermion representation. We find that the dilaton mass remains tied to the dynamical fermion mass scale. It is also of interest that one may justify an arbitrarily small $\beta$-function to all orders in $\alpha$ in the limit of large $C_{2}$ and small number of flavors [6]. The $\beta$-function is then small since chiral symmetry breakıng is occurring at small $\alpha$. It is in this type of limit that the results of this paper are partıcularly striking.

We will work within the standard ladder approximation. But our basic result concerning the high energy contribution to the dilaton mass is not, we believe, just a special consequence of this approximation. One of us has argued [6] that the general form of $k \Sigma(k)$ for small $\beta$ expected beyond the ladder approximation is similar to that obtained in the ladder approxımation. It is the tendency for the peak of the function $k \Sigma(k)$ to occur at ever increasing momentum scales as $\beta$ decreases which is responsible for the basic result.

The dilaton mass is obtained by notıng the behavior of the effective action under scale transformations. Our result appears somewhat surprising to the extent that the scale breaking in the effective action apparently vanishes as $\beta$ vanishes. But the effective action is a functional of $\Sigma(k)$ and when minimized the preferred point in function space does turn out to be characterized by large scale breaking, for arbitrarily small but nonzero $\beta$. That is, upon minimization, the integrals in the effective action always receive support over such a large range of momentum that it is never a good approximation to set the coupling $\alpha(k)$ equal to a constant.

And on the other hand if the coupling actually was constant $(\beta=0)$ then there is no local minimum of the effective action, unless an ultraviolet cutoff is imposed [10]. But then it is the ultraviolet cutoff which breaks scale invarrance and which ensures a large dilaton mass $[8,9]$.
We shall obtain our results via an analytical treatment of the linearized ladder Schwinger-Dyson (SD) ladder, makıng use of the observation in ref. [11] that the solution $\Sigma(k)$ is related to a Whittaker function. And we shall derive the appropriate infrared boundary condition directly from the linearization procedure (our boundary condition is different to that used in ref. [11]). Ref. [5] shows that the $\Sigma(k)$ so obtained agrees quite well with a numerical analysis of the nonlinear equation.

We begin by considering the effective action for a theory with $n$ flavors of massless fermions coupled to a non-abelian gauge field. The fermions are in a representation $\mathrm{r}$ of the gauge group $\mathrm{SU}(N)$ with dimension $d(\mathrm{r})$ and quadratic Casimir $C_{2}(\mathrm{r})$. In Landau gauge and in euclidean space we consider the effective action $\Gamma$ to two-loop order and include as well higher order diagrams responsible for the running coupling $\alpha(k)[12,4]$.

$$
\begin{aligned}
\Gamma & =\frac{n d(\mathrm{r})}{8 \pi^{2}} \int_{0}^{\infty} \mathrm{d} p p^{3} \\
& \times\left[\frac{4 \Sigma^{2}(p)}{p^{2}+\Sigma^{2}(p)}-2 \ln \left(\frac{p^{2}+\Sigma^{2}(p)}{p^{2}}\right)\right] \\
& -\frac{3 n d(\mathrm{r}) C_{2}(\mathrm{r})}{8 \pi^{3}} \int_{0}^{\infty} \mathrm{d} p \frac{p^{2} \Sigma(p)}{p^{2}+\Sigma^{2}(p)} \int_{0}^{\infty} \mathrm{d} k \frac{k^{2} \Sigma(k)}{k^{2}+\Sigma^{2}(k)} \\
& \times \min \{k / p, p / k\} \alpha(\max \{p, k\}) .
\end{aligned}
$$

We follow the derivation of the dilaton mass in ref. [8] except that now we do not use an ultraviolet cutoff. The method consists of examining the behavior of $\Gamma(\rho)$ obtained by making a scale transformation of the order parameter $\Sigma(p) \rightarrow \mathrm{e}^{\rho} \Sigma\left(\mathrm{e}^{-\rho} p\right)$. If the dilaton is considered to be the pseudo-Goldstone boson of spontaneously broken scale invariance, then $\mathrm{d}^{2} \Gamma(\rho) /\left.\mathrm{d} \rho^{2}\right|_{\rho=0}=m_{\sigma}^{2} f_{\sigma}^{2}$, where $m_{\sigma}$ is the dilaton mass, and $f_{\sigma}$ is the dilaton decay constant. This is analogous to finding the pion mass by noting how the vacuum energy changes when $\langle\bar{\psi} \psi\rangle$ undergoes a chiral transformation.

The nontrivial $\rho$ dependence arıses from the second term in (1) and thus $\Gamma(\rho)$ takes the form

$\Gamma(\rho)=\mathrm{e}^{4 \rho}[c-f(\rho)]$, 
where $c$ is independent of $\rho$ and $f(\rho)$ may be written as

$$
\begin{aligned}
& f(\rho)=\frac{3 n d(\mathrm{r}) C_{2}(\mathrm{r})}{4 \pi^{3}} \int_{0}^{\infty} \mathrm{d} k \frac{k \Sigma(k) \alpha\left(\mathrm{e}^{p} k\right)}{k^{2}+\Sigma^{2}(k)} \\
& \times \int_{0}^{k} \mathrm{~d} \rho \frac{p^{3} \Sigma(p)}{p^{2}+\Sigma^{2}(p)} .
\end{aligned}
$$

Using the fact that $\mathrm{d} \Gamma(\rho) /\left.\mathrm{d} \rho\right|_{\rho=0}=0$ we find

$$
\begin{gathered}
m_{\sigma}^{2} f_{\sigma}^{2}=\mathrm{d}^{2} \Gamma(\rho) /\left.\mathrm{d} \rho^{2}\right|_{\rho=0} \\
=-4 f^{\prime}(0)-f^{\prime \prime}(0) .
\end{gathered}
$$

It is clear that if $\Sigma(k)$ is held fixed then the slower $\alpha(k)$ varies with momentum, the smaller $m_{\sigma}$ becomes. But of course $\Sigma(k)$ does depend on how $\alpha(k)$ evolves and it is this dependence to which we now turn. The equation of motion $\Delta \Gamma / \delta \Sigma=0$ is just the ladder SD equation with the running coupling inserted:

$\Sigma(a)=\frac{\lambda(a)}{a} \int_{0}^{a} \mathrm{~d} b \frac{b \Sigma(b)}{b+\Sigma^{2}(b)}+\int_{a}^{\infty} \mathrm{d} b \frac{\lambda(b) \Sigma(b)}{b+\Sigma^{2}(b)}$,

with

$\lambda(a)=\alpha(a) / 4 \alpha_{\mathrm{c}}, \quad \alpha_{\mathrm{c}}=\pi / 3 C_{2}(\mathrm{r})$,

and where $a$ and $b$ denote momenta squared.

For an asymptotically free theory the solution $\Sigma(a)$ to (5) turns out to be a monotonically decreasing function of $a$. With this in mind we find it useful to define the scale $\kappa$ satisfying $\Sigma\left(\kappa^{2}\right)=\kappa$. For $b<\kappa^{2}$ in (5) the denominators are dominated by $\Sigma(b)$ and this damps the integrals in the infrared. To study (5) analytically we will linearize it by replacing $b+\Sigma^{2}(b)$ by $b$ in the denominators and by introducing an infrared cutoff at $\kappa^{2}$.

We are justified in making this approximation in the case of a slowly varying coupling. In this case the obtained solution implies that the action $\Gamma$ recelves most of its contribution from momentum scales much larger than $\kappa$. This indicates that the solution $\Sigma(a)$ for $a>\kappa^{2}$ is relatively insensitive to the manner in which the integrals in (5) are damped in the infrared. And we will find that most of the contribution to the dilaton mass integral also comes from large momenta.

We shall therefore replace (5) by the following.
$\Sigma(a)=\frac{\lambda(a)}{a} \int_{\kappa^{2}}^{a} \mathrm{~d} b \Sigma(b)+\int_{a}^{\infty} \mathrm{d} b \frac{\lambda(b) \Sigma(b)}{b}$,

$\Sigma\left(\kappa^{2}\right)=\kappa$.

Note that these equations must determine $\kappa$ as well as $\Sigma(a)$. The integral equation may now be converted into a differential equation

$\Sigma^{\prime \prime}(a)-\frac{(\lambda / a)^{\prime \prime}}{(\lambda / a)^{\prime}} \Sigma^{\prime}(a)-(\lambda / a)^{\prime} \Sigma(a)=0$,

with

$\Sigma\left(\kappa^{2}\right)=\kappa, \quad \Sigma^{\prime}\left(\kappa^{2}\right)=0$.

Note the new boundary condition. We shall also be requiring that the solution has the appropriate asymptotic behavior.

For asymptotically free theories we are motivated to parameterize $\lambda$ by

$\lambda(x)=A / 2 x, \quad x=\ln \left(a / A^{2}\right)$.

This corresponds to $k \partial_{k} \alpha(k)=-b \alpha^{2}(k)$ with $A \equiv 1 / b \alpha_{\mathrm{c}}$. (This lowest order result may actually be justified to all orders in $\alpha$, for the $\alpha$ relevant for the chiral symmetry breaking, in the limit $C_{2}(\mathrm{r}) \gg N$ and $C_{2}(\mathrm{r}) n \ll N^{3} / d(\mathrm{r})[6]$.) If we change variables in (9), use (11), and define [11]

$G(x)=\left(x / x_{0}\right)^{1 / 2} \exp \left[\left(x-x_{0}\right) / 2\right] \Sigma(x) / \kappa$,

with $x_{0} \equiv \ln \left(\kappa^{2} / \Lambda^{2}\right)$, we obtain

$$
\begin{aligned}
& G^{\prime \prime}(x)+\frac{G^{\prime}(x)}{x(x+1)}+\left(\frac{-1}{4}+\frac{A-1}{2 x}+\frac{A-\frac{1}{2}}{2 x^{2}}\right) \\
& \quad \times G(x)=0, \\
& G\left(x_{0}\right)=1, \quad G^{\prime}\left(x_{0}\right)=\frac{1}{2}\left(1+1 / x_{0}\right) .
\end{aligned}
$$

Of interest are theories with slowly varying coupling constants, i.e. theories where $A$ is large. But we will find that chiral symmetry breakıng occurs when the coupling is close to the critical coupling, $2 A / x_{0}=\alpha\left(x_{0}\right) / \alpha_{\mathrm{c}} \approx 1$. Thus since we need only consider $x \geqslant x_{0} \gtrsim A$ we may neglect certain terms in (12) and consider instead

$G^{\prime \prime}(x)+\left(\frac{-1}{4}+\frac{A-1}{2 x}\right) G(x)=0$.

Eq. (14) was obtained in ref. [11] where it was 
noted that it is a special case of Whittaker's equation [13]:

$W^{\prime \prime}(x)+\left(\frac{-1}{4}+\frac{\gamma}{x}+\frac{\left(\frac{1}{4}-\mu^{2}\right)}{x^{2}}\right) W(x)=0$.

The general solution to this equation may be expressed as a linear combination of the Whittaker function $W_{\gamma, \mu}(x)$ and the linearly independent function $W_{-\gamma \mu}(-x)$. Since we are interested in large $x$ we may neglect the $1 / x^{2}$ terms as before, as long as $\mu^{2}-\frac{1}{4}$ is not too large. This gives us some freedom in choosing $\mu$. In any case it is fortunate that we may neglect the $1 / x^{2}$ term in eq. (12), since its large and positive coefficient cannot be reproduced by Whittaker's equation.

It is well known that the asymptotic form of the spontaneous chiral symmetry breaking solution to the SD equation in an asymptotically free theory is $\Sigma(x) \rightarrow \mathrm{e}^{-r} x^{A / 2-1}$ as $x \rightarrow \infty$ [14]. This is just what follows from $G(x)=W_{(A-1) / 2, \mu}(x)$. On the other hand $G(x)=W_{-\{A-1\} / 2, \mu}(-x)$ yields the asymptotic behavior of the explicit chiral symmetry breaking solution [14]: $\Sigma(x) \rightarrow x^{-1 / 2}$ as $x \rightarrow \infty$. The existence of an ultraviolet cutoff will in general require a linear combination of these two solutions. But in our case of no ultraviolet cutoff only the first solution gives the correct asymptotic behavior.

We now have the function form of the self-energy

$\Sigma(x) \propto x^{-1 / 2} \mathrm{e}^{-\gamma / 2} W_{(4-1) / 2 \mu}(x)$.

We may relate this to the degenerate hypergeometric function via the identity

$W_{\gamma, \mu}(x)=\mathrm{e}^{-1 / 2} x^{\mu+1 / 2} U\left(\mu-\gamma+\frac{1}{2}, 1+2 \mu, x\right)$

and obtain

$\Sigma(x) \propto \mathrm{e}^{-\backslash} x^{\mu} U(-A / 2+1+\mu, 1+2 \mu, x)$.

$x_{0}$ is determined from the boundary condition in (10), $\Sigma^{\prime}\left(x_{0}\right)=0$. For $x>x_{0}$ we require that $\Sigma(x)$ monotonically decrease towards zero (solutions with nodes will give a larger action). Thus $x_{0}$ is the largest $x$ at which the function on the RHS of (18) has a local maximum. We first wish to show as claimed above that $x_{0} \gtrsim A$ for large $A$. We may do this by noting that $U(-n, m+1, x)$ with $n$ and $m$ integers is simply related to the associated Laguerre polynomial $U(-n, m+1, x)=(-1)^{n} n ! L_{n}{ }^{(m)}(x)$. Then $x_{0}$ must be larger than the largest zero of $L_{A / 2-1-\mu}{ }^{(2 \mu)}(x)$ [15].

$$
\begin{aligned}
x_{0} & >x_{\text {zего }} \\
& \approx\left[(2 A-2)^{1 / 2}-1.85575(2 A-2)^{-1 / 6}\right]^{2} .
\end{aligned}
$$

This justifies our neglect of $1 / x^{2}$ terms in (12).

A direct determination of $x_{0}$ shows that $2 A-x_{0}$ is positive and increases with increasing $A$. Thus it is not quite correct to say that chiral symmetry breaking occurs at the "critical" coupling $\alpha(2 A)=\alpha_{\mathrm{c}}$. On the other hand $2 A-x_{0}$ does not grow as fast as $A$ since (19) indicates that $\alpha\left(x_{0}\right) / \alpha_{\mathrm{c}}=2 A / x_{0} \rightarrow 1+$ as $A \rightarrow \infty$.

In the following we shall choose $\mu=0$ for convenience. We also normalize our solution according to $\Sigma\left(x_{0}\right)=\kappa$ to obtain

$\Sigma(x)=\kappa \exp \left[\left(x_{0}-x\right)\right] \frac{U(1-A / 2,1, x)}{U\left(1-A / 2,1, x_{0}\right)}$.

We may now return to finding the dilaton mass as given by eq. (4). Neglecting terms down by $1 / A$ and linearizing as before we find

$$
\begin{aligned}
& m_{\sigma}^{2} f_{\sigma}^{2} \approx\left[\operatorname{And}(\mathrm{r}) \kappa^{2} / \pi^{2}\right] \\
& \quad \times \int_{x=0}^{\infty} \mathrm{d} x \frac{\Sigma(x)}{x^{2}} \int_{x_{0}}^{\mathrm{r}} \mathrm{d} y \exp \left(y-x_{0}\right) \Sigma(y) .
\end{aligned}
$$

Using the identity $U^{\prime}(a, b, z)=-a U(a+1, b+1, z)$, the first integral may be done giving

$$
\begin{aligned}
& m_{\sigma}^{2} f_{\sigma}^{2} \approx\left[2 n d(\mathrm{r}) \kappa^{4} / \pi^{2}\right] \\
& \quad \times \int_{x}^{\infty} \mathrm{d} x \frac{\exp \left(x_{0}-x\right) U(1-A / 2,1, x)}{x^{2} U\left(1-A / 2,1, x_{0}\right)^{2}} \\
& \quad \times\left[U(-A / 2,0, x)-U\left(-A / 2,0, x_{0}\right)\right] .
\end{aligned}
$$

This integral is not very sensitive to the behavior of the integrand near $x_{0}$ (where our approximations are suspect), since the integrand peaks at a point $\hat{x}$ such that $\hat{x}-x_{0}$ grows with $A$.

Numerically it is found that the integral in (22) approaches $\frac{1}{2}$ as $A$ grows large, as shown in fig. 1 . (And as expected this is also true for nonzero $\mu^{2} \ll A$.) We know of no a prior1 reason to explain this surprising tendency for the integral to approach a constant. But it implies that

$m_{\sigma}^{2} f_{\sigma}^{2} \rightarrow n d(\mathrm{r}) \kappa^{4} / \pi^{2}, \quad$ for large $A$. 


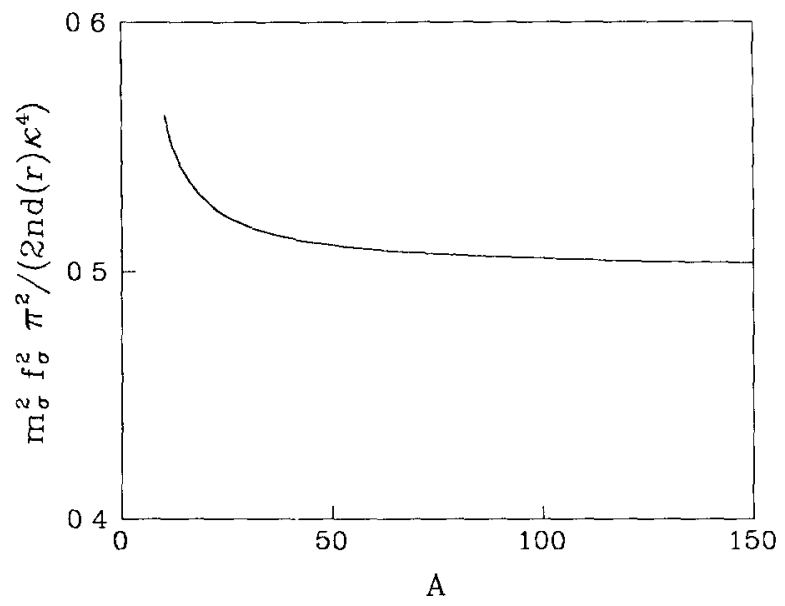

Fig 1 The $A$ dependence of the quantity $m_{\sigma}^{2} f_{\sigma}^{2}$ where $A \equiv 3 C_{2}(\mathrm{r}) / \pi b$ and $b$ is defined by $k \partial k \alpha(k)=-b \alpha(k)^{2}$

Whether or not the integral does in fact tend exactly to $\frac{1}{2}$ as $A \rightarrow \infty$, it is clear that $m_{\sigma}^{2} f_{\sigma}^{2}$ does not have the $1 / A$ dependence naively expected. Instead, at least for large $A, m_{\sigma}^{2} f_{\sigma}^{2}$ has surprisingly little dependence on $A$.

The dilaton decay constant $f_{\sigma}$, like the pion decay constant $[4,5]$, is also expected to be relatively insensitive to $A$ and to be of order $\kappa$. Thus the dilaton mass is tied quite closely to the dynamical fermion mass, with the latter being of order $\kappa \approx \Sigma(0)$. The case [6] $C_{2}(\mathrm{r}) \gg N$ and $C_{2}(\mathrm{r}) n \ll N^{3} / d(\mathrm{r})$ implies both a large $A$ and a confinement scale much smaller than $\kappa$. This shows that the dilaton mass we have calculated is independent of confinement physics.

In actual practice, for a technicolor theory, if $A$ is too large then new "sideways" physics will start to influence the high energy behavior of $\Sigma(k)$. This new physics may act as a physical ultraviolet cutoff, as considered in ref. [8]. But we may now conclude that there is never a light dilaton, no matter what the very high energy behavior of the theory is like.

We have found that even though the apparent measure of explicit scale breaking, $\beta(\alpha(k))$, is small for any $k$ relevant for the spontaneous chiral (and scale) symmetry breaking, the pseudo-Goldstone boson associated with this apparent approximate scale symmetry does not have a small mass relative to the dynamical fermion mass. The resolution to this paradox lies in the fact that effective action is a func- tional of the order parameter $\Sigma(k)$. At the preferred point in function space the function $k \Sigma(k)$ does not decrease until high momentum, with the result that the effective action always samples $\alpha(k)$ over a sufficiently large range of $k$ such that the variation of $\alpha(k)$ is always significant. It is never a good approximation to set $\alpha(k)$ equal to a constant in the effective action governing chıral symmetry breaking for an asymptotically free theory with no ultraviolet cutoff.

This research was supported in part by the Natural Sciences and Engineering Research Council of Canada.

\section{References}

[1] B. Holdom, Phys. Rev D 24 (1981) 1441, Phys Lett B 150 (1985) 301

[2] T Akıba and T. Yanagıka, Phys Lett B 169 (1986) 432.

[3] K. Yamawakı, M Bando and K Matumoto, Phys Rev. Lett 56 (1986) 1335, Phys Lett B 178 (1986) 308

[4] T Appelquist, D Karabalı and L C.R Wijewardhana, Phys Rev Lett 57 (1986) 957,

T. Appelquist and L.C.R Wijewardhana, Phys. Rev D 35 (1987) 774; D 36 (1987) 568

[5] B Holdom, Phys. Lett B 198 (1987) 535

[6] B Holdom, Chiral hierarchies beyond the ladder approximation, University of Toronto preprint UTPT-87-12 (1987)

[7] W Bardeen, C Leung and S Love, Phys. Rev Lett 56 (1986) 1230; Nucl Phys. B 273 (1986) 649,

T. Clark, C Leung and S Love, Phys Rev. D 35 (1987) 998

[8] B Holdom and J. Terning, Phys Lett B 187 (1987) 357

[9] V Gusynin and M. Miransky, Nonperturbatıve scale anomaly and dilaton in gauge field theories, Kıev preprint ITP-87-58E (1987)

[10] T Maskawa and $H$ Nakajıma, Prog Theor. Phys 52 (1975) 1326, 54 (1975) 860,

R Fukuda and T Kugo, Nucl Phys. B 117 (1976) 250

[11] M Bando, T Morozum1, H So, K. Yamawak1, Phys Rev Lett 59 (1987) 389.

[12] M Peskın, in: Recent advances in field theory and statıstical mechanics, eds J. Zuber and R Stora, Les Houches Summer School Proc, Vol 39 (North-Holland, Amsterdam, 1984)

[13] Eg. M Abramowitz and I Stegun, eds, Handbook of mathematical functions (Dover, New York, 1965)

[14] H.D. Politzer, Nucl Phys. B 117 (1976) 397;

P I Fomın, VP Gusynin, VA Miransky and Yu A. S1tenko, Rev Nuovo Cimento 6 (1983) 1

[15] G. Szego, Orthogonal polynomials (American Mathemat1cal Society, New York, 1959) 